\title{
THERMONUCLEAR RUNAWAY MODEL
}

\author{
Warren M. Sparks \\ Los Alamos National Laboratory \\ G. Siegfried Kutter \\ National Science Foundation \\ Sumner Starrfield \\ Arizona State University and \\ Los Alamos National Laboratory \\ and \\ James W. Truran \\ University of Illinois
}

\section{INTRODUCTION}

The nova outburst requires an energy source that is energetic enough to eject material and is able to recur. The Thermonuclear Runaway (TNR) model, coupled with the binary nature of nova systems, satisfies these conditions. The white dwarf/red dwarf binary nature of novae was first recognized as a necessary condition by Kraft (1963, 1964, and these conference proceedings). The small separation characteristic of novae systems allows the cool, red secondary to overflow its Roche lobe. In the absence of strong, funneling magnetic fields, the angular momentum of this material prevents it from falling directly onto the primary, and it first forms a disk around the white dwarf. This material is eventually accreted from the disk onto the white dwarf. As the thickness of this hydrogen-rich layer increases, the degenerate matter at the base reaches a temperature that is high enough to initiate thermonuclear fusion of hydrogen. Thermonuclear energy release increases the temperature which in turn increases the energy generation rate. Because the material is degenerate, the pressure does not increase with temperature, which normally allows a star to adjust itself to a steady nuclear burning rate. Thus the temperature and nuclear energy generation increase and a TNR results. When the temperature reaches the Fermi temperature, degeneracy is lifted and the rapid pressure increase causes material expansion. The hydrogen-rich material either is ejected or consumed by nuclear burning, and the white dwarf returns to its pre-outburst state. The external source of hydrogen fuel from the secondary allows the whole process to repeat.

\section{II . THE DEVELOPMENT OF A TNR}

The temperature evolution provides significant information about the development of a TNR. Figure 1 shows the temperature structure of the hydrogen-rich shell on a white dwarf at various times during its evolution. It is from an equilibrium model with $\mathrm{H}$ initially in place (see $\$ I I I \mathrm{~A}$ ), and its behavior is characteristic of all models. Curve 1 on the graph is the initial temperature distribution of the envelope. It is determined by the intrinsic white dwarf's luminosity. Curve 2 represents the distribution when the proton capture onto CNO nuclei dominates over the p-p reactions at $\sim 2 \times 10^{7} \mathrm{~K}$. The time between these first two curves is usually many thousands of years and is very strongly dependent on the white dwarf's intrinsic 
luminosity and weakly dependent on the CNO abundance of the envelope's matter and the white dwarf's mass. The next significant event, as the temperature continues to rise, occurs at $\sim 3 \times 10^{7} \mathrm{~K}$ when convection begins in the region above the maximum temperature (curve 3 ). This convective region continues to grow outward until it reaches the surface layers near the time of peak temperature. When the temperature (curve 4) reaches $10^{8} \mathrm{~K}$ two significant circumstances occur. First, the temperature exceeds the Fermi temperature and the electron degeneracy is lifted. At this time the pressure increases rapidly and hydrodynamics must be included in the calculations. Second, the proton capture rates on the CNO nuclei become shorter than the $B^{+}$ decay rates of the temporary reaction products of the $\mathrm{CNO}$ bicycle $\left({ }^{13} \mathrm{~N},{ }^{14} \mathrm{O},{ }^{15} \mathrm{O}\right.$, and $\left.{ }^{17} \mathrm{~F}\right)$. Thus, the $\mathrm{B}^{+}$- unstable nuclei and their rates must be included both in the nuclear reaction network and in the energy generation calculation (Starrfield et al. 1972). As the temperature increases, the CNO reactions are controlled by the $B^{+}$decay rates, and the energy generation rate becomes independent of temperature and density and dependent only on the CNO isotopic abundances.

The last curve (\#5) corresponds to the time of peak temperature, which is reached only a few seconds after curve 4 . The dynamic time scale is now of the order of one second while the nuclear burning time scale is given by $\mathrm{c}_{\mathrm{p}} \mathrm{T} / \varepsilon_{\text {nuc }}$. If the $\mathrm{CNO}$ abundances are solar then the nuclear burning time scale is also of the order of one second. This allows the envelope to expand before a strong TNR can develop. Under these conditions little or no material is ejected by the expansion. The now rekindled hydrogen burning shell source may eject the envelope by radiation pressure (or common envelope interaction) and produce a slow nova (Sparks et al. 1978). If the initial CNO abundances are increased above solar, the nuclear burning time scale becomes shorter than the dynamic time scale. For large CNO overabundances the TNR becomes strong enough to eject a portion of the hydrogen-rich envelope and produce a fast nova (Starrfield et al. 1978). The peak temperature is also strongly dependent on the CNO abundances. At peak temperature the convective time scale is of the order of $100 \mathrm{sec}$. This is approximately the decay time scale of the $\mathrm{B}^{+}$- unstable nuclei. Therefore, a sizeable fraction of the $\mathrm{B}^{+}$- unstable nuclei will be convected to the outer layers before they decay. When they decay in the outer layers, they provide an additional energy source for ejection at a relatively low gravity. This complexity of physical phenomena shows why it is necessary to treat hydrodynamics, time-dependent convection, and nuclear physics correctly.

The energy rates are a useful diagnostic tool to understand the TNR. The local energy conservation equation can be written in the form (Kutter and Sparks 1974, 1980):

$$
\frac{\partial}{\partial \mathrm{t}}\left\langle\frac{\mathrm{u}^{2}}{2}-\frac{\mathrm{GM}}{\mathrm{r}}+\mathrm{E}_{\mathrm{int}}\right\rangle+\frac{\partial \mathrm{L}_{\mathrm{ph}}}{\partial \mathrm{m}}+\frac{\partial}{\partial \mathrm{m}}\left(4 \pi \mathrm{r}^{2} \mathrm{uP}\right)=\varepsilon_{\mathrm{nuc}}
$$

$\mathrm{L}_{\mathrm{ph}}$ includes the transport of energy by both radiative diffusion and convection. We label the terms on the left side of equation (1) $\varepsilon_{\mathrm{KIN}}, \varepsilon_{\mathrm{GRAV}}, \varepsilon_{\mathrm{INT}}$, and $\varepsilon_{\mathrm{MECH}} \cdot \varepsilon_{\mathrm{NUC}}$ is always positive and represents the nuclear energy source. The other terms of equation (1) may be either positive or negative, depending on whether energy is being stored (sink of energy) or released (source of energy), respectively. At all times and locations in the envelope, the sum of these terms must equal the nuclear energy source. By monitoring these terms, we can determine the accuracy of the code. 
Figure 2 shows these energy rates as a function of mass during the early evolutionary stages of an accreting $1.0 \mathrm{M}_{\odot}$ white dwarf (Kutter and Sparks 1980). The nuclear energy source is small and gravity is the main energy source for the photon, internal and mechanical energy sinks. Basically, the accreting hydrogen envelope is being compressed and heated under its own weight. Figure 3 shows a later stage of evolution when the convective region is extending outward. The strength of the nuclear energy source has increased and the resulting pressure increase has changed the motion within the envelope from slowly contracting to slowly expanding. This is indicated by the positive sign for the gravitational energy throughout the envelope. Convective flux is the main source of energy above the nuclear energy generation region and mechanical flux the main source in the outer regions. These fluxes are carrying energy from the nuclear energy generation region and depositing it farther out. The leading edge of the convective region is indicated by a sharp drop of both the convective flux (source) and the internal energy (sink).

The energy rate distribution near peak nuclear burning is presented in Figure 4. The convective region extends all the way to the surface and the convective flux is the main source of energy to the outer layers. The internal energy is now a source of energy because of the expansion. The nuclear energy source now extends all the way to the surface because the convective time scale is short enough so that some of the $\mathrm{B}^{+}-$unstable nuclei reach the surface before they decay. Almost all of this energy is going into the lifting (i.e., gravitational energy) of material. As the convective flux decreases the $\mathrm{B}^{+}$- unstable nuclei became an important energy source for the outer layers.

\section{COMPUTATIONAL MODELS}

The main computational parameters that affect the strength of a TNR are the pressure in the energy generation region and its composition, specifically, the HCNO nuclei. The nuclear energy rate is proportional to the product of the abundance of $\mathrm{H}$ and the $\mathrm{CNO}$ isotopes. Normally the abundance of $\mathrm{H}$ is large and does not change over the critical stages of the TNR. Thus the CNO abundances are through their catalytic effect the controlling factor in the energy generation rate, especially when the $\mathrm{B}^{+}$decay rates dominate the burning cycle (see Fig 5). The pressure confines the TNR allowing the temperature and energy generation to reach high values. Analytical studies by Fujimoto $(1982 \mathrm{a}, \mathrm{b})$ give a critical pressure $\left(\log \mathrm{P}_{\mathrm{c}} \cong\right.$ 20.0) necessary for mass ejection, while numerical simulations indicate $\log P_{c} \cong 19.3$ (Truran and Livio 1986). The pressure is determined by the overlying envelope mass and the gravity. The gravity is specified by the mass and radius of the white dwarf. Since the radius of a white dwarf depends mainly on its mass, it follows that the gravity depends only on the white dwarf's mass. We will now consider the various types of nova models calculated to date to see what controls the strength of the TNR. In particular, we will investigate what roles the input parameters and the physics play.

\section{A. H-In-Place Equilibrium Models}

The first realistic hydrodynamic studies of novae involved models with the hydrogen-rich envelope initially in place and in hydrostatic and thermal equilibrium on the white dwarf (Starfield et al. 1972; Prialnik et al. 1978; McDonald 1979). The observed features of the classical nova--observed visual light curve, velocity curve, constant bolometric luminosity and shift of the wavelength of peak emission--were simulated by these models (Sparks et al. 1976; Starfield et al. 1978). The mass and intrinsic luminosity of 
the white dwarf and the mass and composition of the envelope were input parameters for these models. These input parameters determined the pressure and composition of the nuclear energy generation region and, thus, the strength of the TNR. Whether or not energy is allowed to flow into the white dwarf's core (below the hydrogen-rich envelope) has only a small effect on the depth and thus on the strength of the TNR. For a specified white dwarf mass, the strength of the TNR depends mainly on the input envelope mass and composition. The selection of the envelope mass was guided by hydrostatic accreting white dwarf models (Giannone and Weigert 1967; Taam and Faulkner 1975). At an early stage of modeling it was discovered that the abundance of the $\mathrm{CNO}$ nuclei had to be enhanced above solar to get a strong TNR (Starrfield et al. 1972). Although it was suspected that this enhancement came from a mixing up of white dwarf core material, the nature and efficiency of the mixing process were unknown and the CNO enhancement was artificially set.

\section{B. Accretion Models}

The next logical improvement in nova calculations was an accreting white dwarf model (Nariai et al. 1980; Kutter and Sparks 1980; McDonald 1980; Prialnik et al. 1982). In these models the white dwarf's mass and intrinsic luminosity, the accretion rate and the internal energy and composition of the accreting material are the input parameters. The envelope mass is not an input parameter but a complex function of the accretion rate and the internal energy of the accreting material and the physics of radiation losses and compressional heating during accretion. It is also weakly dependent on the composition of the accreting matter. This allows a more realistic and satisfying method of determining the envelope mass. These models also give general agreement with the same observations as the $\mathrm{H}$-in-place equilibrium models did. In addition, we can make a more valid comparison of the calculated ejected mass with observation. This also is in good agreement.

Three problems are evident in the calculations. First, the mechanism of the enhancement of the CNO nuclei was not understood. The second is shown in a plot of accretion rate verses white dwarf mass (Figure 6). The location on this graph of the observed novae (Patterson 1984) are indicated by asterisks, while the models that eject nova-like material (Prialnik et al. 1982; Starrfield et al. 1985) are indicated by dots. These models are more massive and accrete at a slower rate than the observed values. Models which have values similar to those observed produce weak TNRs that do not eject material rapidly or look like a nova. They tend to expand and then settle down to a red giant configuration or to appear like a symbiotic novae (Livio $e t$ al. 1989). Third, most accretion models have assumed that the accreted material has the same internal energy as the outer layers when it comes to rest. Recently, Shaviv and Starrfield (1987) and Regev and Shara (1989) have shown that using more realistic boundary conditions leads to a higher accretion energy and an earlier and weaker TNR. This will cause the viable models in Figure 6 to move downward to even lower accretion rates and thus, aggravate the second problem.

\section{Accretion Models With Mixing}

Observations of nova ejecta offer ample evidence for the mixing of white dwarf material with the accreted material (Truran and Livio 1986; Sparks et al. 1988). In an attempt to solve the problems of the enhancement of CNO nuclei in nova ejecta and less massive white dwarfs producing novae, accretion models with mixing have been simulated. We arbitrarily divide these models into the categories of long-term 
and short-term mixing. Long-term mixing includes those mixing processes which persist throughout the accretion process. Short-term mixing includes those processes which occur when convection turns on.

1. Long-Term Mixing

a) Diffusion

Diffusion allows the hydrogen-rich accreted material to penetrate into the white dwarf's core material and vice versa. Prialnik and Kovetz (1984) and Kovetz and Prialnik (1985) have evolved hydrostatic 0.9 and $1.25 \mathrm{M}_{\odot}$ models with fine-meshed zones to accurately determine the diffusion. Figure 7 shows the resulting composition distribution for a $1.25 \mathrm{M}_{\ominus}$ white dwarf model with an accretion rate of $10^{-12} \mathrm{M}_{\odot} / \mathrm{yr}$. They have found that the TNR occurs below the initial core-envelope interface and that the CNO abundance in the envelope can be increased up to $40 \%$. Because of the use of a hydrostatic code, it is not known which of their models would produce a nova. The range of accretion rates chosen for these diffusion models are much slower than for the non-diffusion accretion models. This is because of the long time scale of diffusion. This separates the models even further from the observations (see Figure 6). Shara et al. (1986) and Prialnik and Shara (1986) have suggested that the white dwarf spends a considerable amount of time in a "hibernation" state. Thus, the actual average accretion would be much lower than the observed accretion rate in agreement with simulations.

b) Accretion-Induced Shear Mixing

As disk material with high angular momentum is accreted onto the white dwarf that initially has little or no rotation, a shear instability develops. Kippenhahn and Thomas (1978) and Kutter and Sparks (1987) show that under the condition of marginal stability a component of the angular momentum gradient is in the radial direction. The accreted material is shear mixed with the white dwarf material and the composition distribution, to first order, is a function of the accreted mass only. The conservation of angular momentum causes rotational energy to be converted into internal energy, and the TNR occurs too soon to produce mass ejection for a $1.0 \mathrm{M}_{\odot}$ model (Sparks and Kutter 1987). Additional short-term shear mixing effects are discussed in $\S I I I 2 \mathrm{c}$ ).

c) Comparison of Diffusion and Shear Mixing

For an accreting white dwarf both diffusion and shear mixing take place simultaneously. In order to determine the regimes where each dominates, we have done the following: A $1.25 \mathrm{M}_{\odot}$ accreting shear mixing white dwarf was evolved until its composition distribution was similar to the D12 diffusion model (see Figure 7) of Prialnik and Kovetz (1984). The composition distribution due to shear mixing is to first order determined by the amount of accreted mass while that due to the diffusion is to first order determined by the accretion time. If we take this accreted mass and divided it by the time then we have a critical accretion rate where the two processes produce similar composition distributions. For accretion rates above this critical value, shear mixing dominates while below it diffusion dominates. For this comparison, the critical accretion rate is $10^{-17} \mathrm{M} \odot / \mathrm{yr}$. If the angular momentum content of the accreting material is reduced by a factor, then this critical accretion rate is increased by that same factor. For example, if the angular momentúm per gram accreted is only $10^{-2}$ of the Keplerian disk angular momentum per gram, then this critical accretion rate is raised to $10^{-15} \mathrm{M}_{\mathcal{\Theta}} / \mathrm{yr}$. Thus it appears that shear mixing dominates except for the case of polar 
accretion in DQ Her and AM Her stars. Livio and Truran (1987) point out some observational difficulties for both the diffusion and shear mixing mechanisms.

2. Short-Term Mixing

a) Convective Overshooting

Convective mixing is an extremely important mechanism in nova modeling and is included in all of the previously discussed models. It carries $\mathrm{B}^{+}$- unstable nuclei to the cooler outer regions and brings fresh nuclear fuel down to the TNR region (Starrfield et al. 1977). In its normal formulation convection does not allow material from the TNR region to be mixed with deeper layers and vice versa. However, convective overshooting will mix these two regions. A number of years, ago two of us (W.M.S. and G. S. K.) simulated convective overshooting in a $1.0 \mathrm{M}_{\odot}$ nova model by allowing the convective elements to cross the convective/non-convective boundary with the calculated convective velocity. These elements then decelerated due to the difference in density between them and their surroundings. This allowed us to estimate how much energy is deposited in the lower regions. It turned out that the energy deposition was not enough to move the TNR region inward appreciably. However, Woosley (1986) evolved a $1.2 \mathrm{M}_{\Theta} \mathrm{CO}$ white dwarf accreting solar composition material with a diffusion coefficient prescription for overshooting. He found that the CNO abundance of the ejected material $\sim 10 \%$. Obviously convective overshooting must be investigated further.

b) Flame Propagation

The TNR region does not develop at the deepest point of hydrogen penetration into the core material for either the diffusion or the accretion-induced shear mixing models. Thus, there is hydrogen fuel below the TNR region. Therefore, in order for the TNR region to move deeper, it is only necessary to ignite the deeper layers. The mathematical formulation of such a process, possibly modeled after flame propagation, is needed. Woosley (1986) suggested this could be in the form of turbulent diffusion similar to the overshoot diffusion.

c) Convection-Induced Shear Mixing

The angular momentum distribution of accretion-induced shear mixing models increases monotonically with radius as shown schematically in Figure 8a. Convection tends to flatten out the angular momentum distribution (see Figure 8b), just as it flattens out the composition distribution, creating a steep angular momentum gradient at the inner and outer boundaries of the convective region. This, in turn, leads to convection-induced shear mixing which causes angular momentum and accreted material to be transported from the outer layers to the deeper layers (see Figure 8c). Exploratory studies by Kutter and Sparks (1989) of this mechanism suggest that it is capable of greatly enhancing the TNR.

Recently two of us (G.S. K. and W.M.S. have evolved a $1.0 \mathrm{M}_{\odot}$ CO white dwarf accreting $4.23 \times 10^{-10} \mathrm{M} \odot$ yr. This model included accretion-induced shear mixing similar to previous models (Sparks and Kutter 1987). In addition, they assumed that convection-induced shear mixing reached marginal stability instantaneously. The resulting TNR was extremely violent with the peak energy generation reaching $2.4 \times 10^{16} \mathrm{erg} /(\mathrm{g}-\mathrm{sec})$. This model ejected about 3 times as much material as was accreted, with velocities up to $3000 \mathrm{~km} / \mathrm{sec}$. For accretion-induced shear mixing, the assumption of 
reaching marginal stability instantaneously is reasonable because of the long time scale for the accretion process. However, for convection-induced shear mixing, this assumption is probably not valid because of the much shorter convective time scale. Therefore, this model is probably too violent, but it does show the potential enhancement of the TNR by this mechanism. A timedependent convection-induced shear mixing method is being formulated.

\section{OBSERVATIONAL EVIDENCE OF TNR}

Although the TNR model has predicted and reproduced many observational nova features (Sparks et al.1976), observational confirmation of a TNR is more difficult. Since $\mathrm{C}$ is more abundant than $\mathrm{N}$ in a solar mix (and presumably in the donor red star companion ), then $\mathrm{C}$ should be more abundant in the nova ejecta unless there has been proton capture onto the CNO nuclei. This becomes an even stronger statement if material is mixed up from a $\mathrm{CO}$ white dwarf core. The $\mathrm{CO}$ white dwarfs that novae initially had roughly 50\% $\mathrm{C}$ and 50\% O (Sparks et al. 1988). For the CNO nuclei, C captures protons most readily and $\mathrm{N}$ the least, so that the higher abundance of $\mathrm{N}$ than $\mathrm{C}$ in all well-observed nova ejecta (Truran and Livio 1986) is a signature of $\mathrm{CNO}$ burning. However, CNO burning does not necessarily mean that a TNR occurred.

Sneden and Lambert (1975) analyzed the CN molecular bands near $4215 \AA$ which appeared in the spectrum of DQ Her near maximum light. They found upper limits on ${ }^{13} \mathrm{C} / \mathrm{C}$ and/or ${ }^{15} \mathrm{~N} / \mathrm{N}$ which indicates that the $\mathrm{CNO}$ burning did not only depend upon the proton capture rates, but also upon the $\mathrm{B}^{+}$decay rates (Caughlan and Fowler 1962, 1972). This means that the temperature was at least $10^{8} \mathrm{~K}$. A TNR is the only known way of reaching such a high temperature in the outer layers of a white dwarf. Unfortunately, lower limits on these ratios were not given. These ratios should be recalculated with synthetic spectra from modern codes.

Additional evidence of a TNR would be the observation of $\gamma$-line emission from the $\mathrm{B}^{+}$- unstable nuclei in nova ejecta (Truran et al. 1978). Hoffman and Woosley (1986) calculated that the decay signal from the ${ }^{22} \mathrm{Na} \gamma$-line may be visible to terrestrial detectors from novae within one kiloparsec. The positron from the decay of $\mathrm{B}^{+}$- unstable nuclei will also produce $\gamma$-rays from the electron-positron annihilation. The prospects of detecting these with satellite $\gamma$-ray spectrometers are discussed by Leising and Clayton (1987).

The TNR model has been universally accepted as the cause of the nova outburst because of its agreement with observations. The mechanism for mixing white dwarf core material with accreted material is still under investigation.

We would like to express our thanks for many useful discussions to Drs. A. Kovetz, R. Kraft, M. Livio, D. Prialnik, M. Shara, G. Shaviv, E. Sion, R. Williams, and S. Woosley. W. Sparks is grateful to the IUE Observatory and the IAU Colloquium No. 122 organizing committee for financial aid. S. Starrfield is grateful to Drs. S. Colgate, A. N. Cox, C. F. Keller, M. Henderson, and K. Meyer for the hospitality of the Los Alamos National Laboratory and a generous allotment of computer time. This work was supported in part by NSF Grants AST85-16173 and AST88-18215 to Arizona State University and AST86-11500 to 
the University of Illinois, by the Institute of Geophysics and Planetary Physics at Los Alamos, by NASA grants to Arizona State University and to the University of Colorado, and by the DOE.

\section{REFERENCES}

Caughlan, G. R., and Fowler, W. A. 1962, Ap. J., 136, 453. 1972 Nature Phys. Sci., 238, 23.

Fujimoto, M. Y.1982a, Ap. J., 257, 752. 1982 b, Ap. J., 257, 767.

Giannone, P., and Weigert, A. 1967, Zs.f. Ap., 67, 41.

Hoffman, R., and Woosley, S. E. 1986, BAAS, 18, 948.

Kippenhahn, R., and Thomas, H.-C. 1978, Astr. Ap., 63, 265.

Kovetz, A., and Prialnik, D. 1985, Ap. J., 291, 812.

Kraft, R. P. 1963, Adv. Astr. and Ap., 2, 43. 1964, Ap. J., 139, 457.

Kutter, G. S., and Sparks, W. M. 1974, Ap. J., 192, 447. 1980, Ap. J., 239, 988. 1987, Ap. J., 321, 386. 1989, Ap. J., 340, 985.

Leising, M. D. , and Clayton, D. D. 1987, Ap. J., 323, 159.

Livio, M., Prialnik, D., and Regev, O. 1989, Ap. J., 341, 299.

Livio, M., and Truran, J. W. 1987, Ap. J., 318, 316.

MacDonald, J. 1979, Ph.D. Thesis, University of Cambridge. 1980, M.N.R.A.S., 191, 933.

Nariai, K., Nomoto, K., and Sugimoto, D. 1980, P.A.S.J., 32, 473.

Patterson, J. 1984, Ap. J. Suppl., 54, 443.

Prialnik, D., and Kovetz, A. 1984, Ap. J., 281, 367.

Prialnik, D., Livio, M., Shaviv, G., and Kovetz, A. 1982, Ap. J., 257, 312.

Prialnik, D., and Shara, M. M. 1986, Ap. J., 311, 172.

Prialnik, D., Shara, M. M., and Shaviv, G. 1978. Astr. Ap., 62, 339.

Regev, O., and Shara, M. M. 1989, Ap. J., 340, 1006.

Shara, M. M., Livio, M., Moffat, A. F. J., and Orio, M. 1986, Ap. J., 311, 163.

Shaviv, G., and Starrfield, S. 1987, Ap. J. (Letters), 321, L51.

Sneden, C., and Lambert, D. L. 1975, M.N.R.A.S., 170, 533.

Sparks, W. S., and Kutter, G. S. 1987, Ap. J., 321, 394.

Sparks, W. S., Starrfield, S., and Truran, J. W. 1976, Ap. J., 208, 819. 1978, Ap. J., 220, 1063.

Sparks, W. S., Starrfield, S. G., Truran, J. W. , and Kutter, G. S. 1988, in Atmospheric Diagnostics of Stellar Evolution: Chemical Peculiarity, Mass Loss, and Explosion, ed. K. Nomoto (Berlin: Springer-Verlag), p. 234.

Starrfield, S., Sparks, W. M., and Truran, J. W. 1985, Ap. J., 291, 136.

Starrfield, S., Truran, J., and Sparks, W. M. 1977, in CNO Isotopes in Astrophysics, ed. J. Audouze (Dordrecht: Reidel), p. 49. 1978. Ap.J., 226, 186.

Starrfield, S., Truran, J. W., Sparks, W. M., and Kutter, G. S. 1972, Ap. J., 176, 169.

Taam, R., and Faulkner, J. 1975, Ap. J., 198, 435.

Truran, J. W., and Livio, M. 1986, Ap. J., 308, 721.

Truran, J. W., Starrfield, S., and Sparks, W. M. 1978, in Gamma Ray Spectroscopy in Astrophysics, ed. T. L. Cline and R. Ramaty, (NASA Technical Memorandum 79619), p. 315.

Woosley, S. E. 1986, in Sixteenth Advanced Course of the Swiss Seciety of Astronomy and Astrophysics, ed. B. Hauck, A. Maeder, and G. Meynel (Geneva: Geneva Observatory), p. 1. 


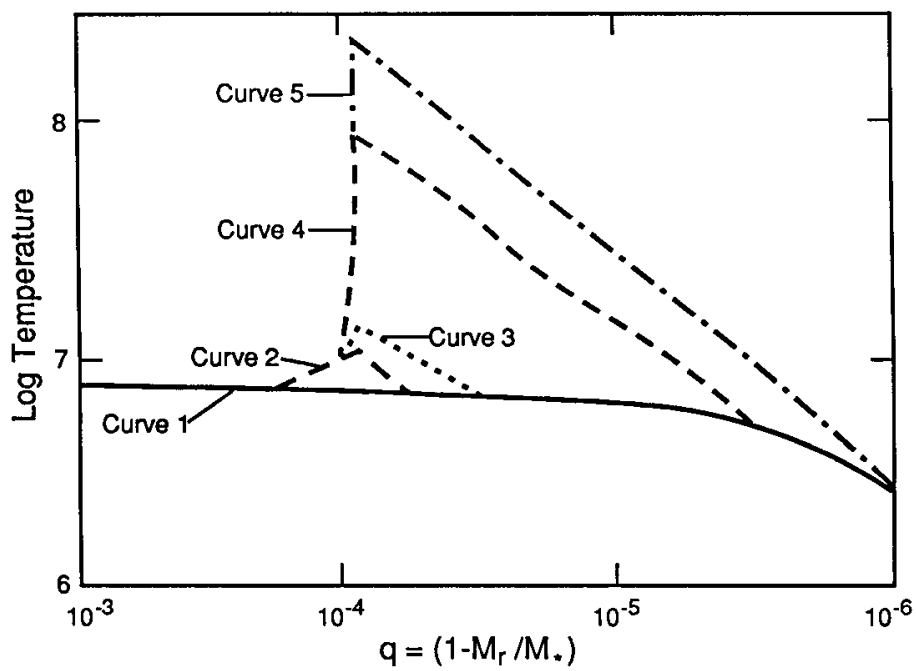

8906852-2-adb

Fig. 1. Log temperature versus mass at various times during the TNR.

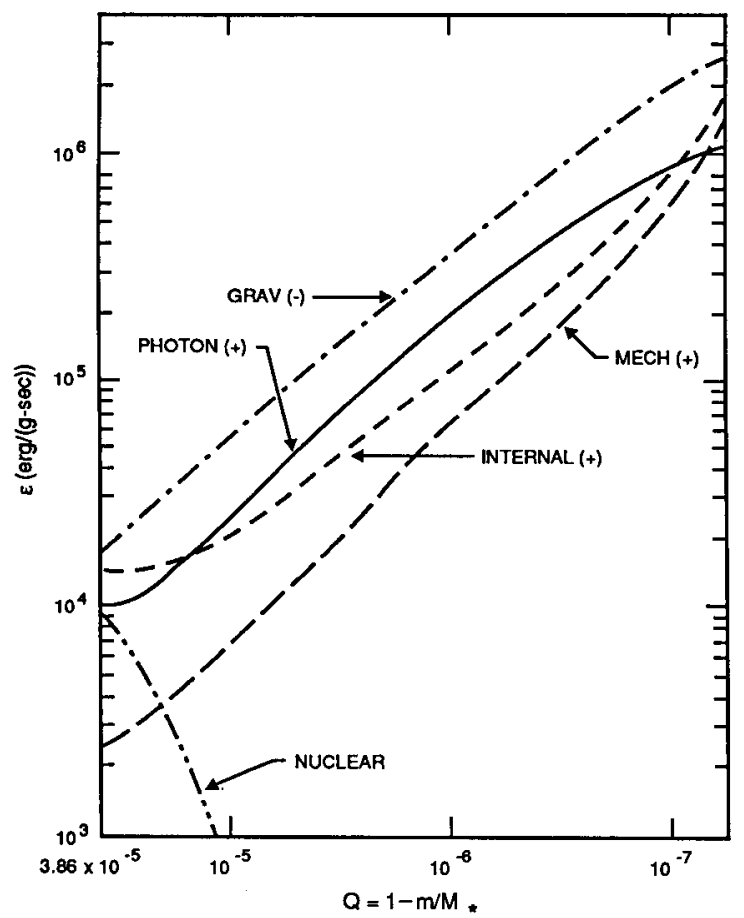

Fig. 2. Energy rates versus mass during the early stages of an accreting white dwarf. 


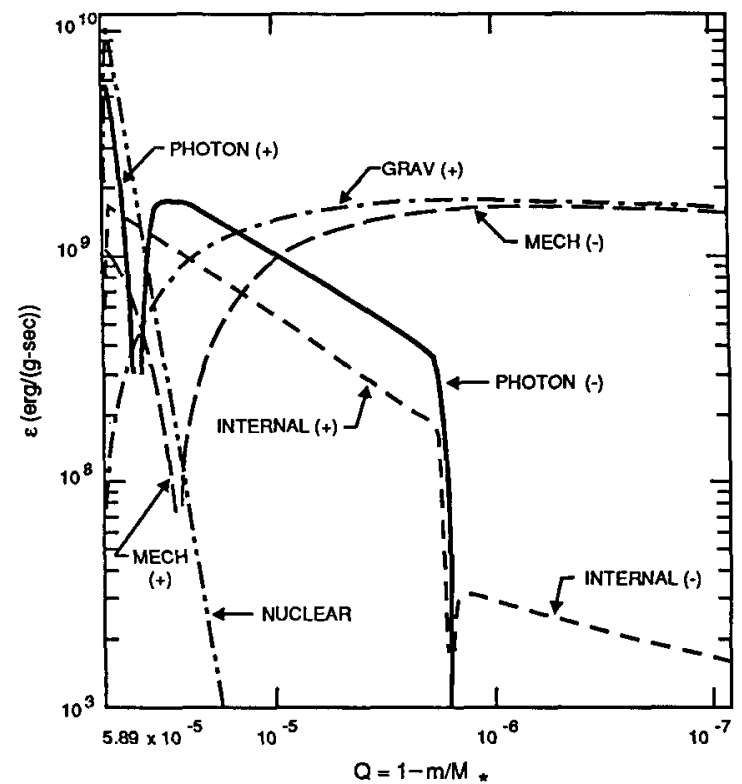

Fig. 3. Energy rates versus mass after the formation of a convective region on an accreting white dwarf.

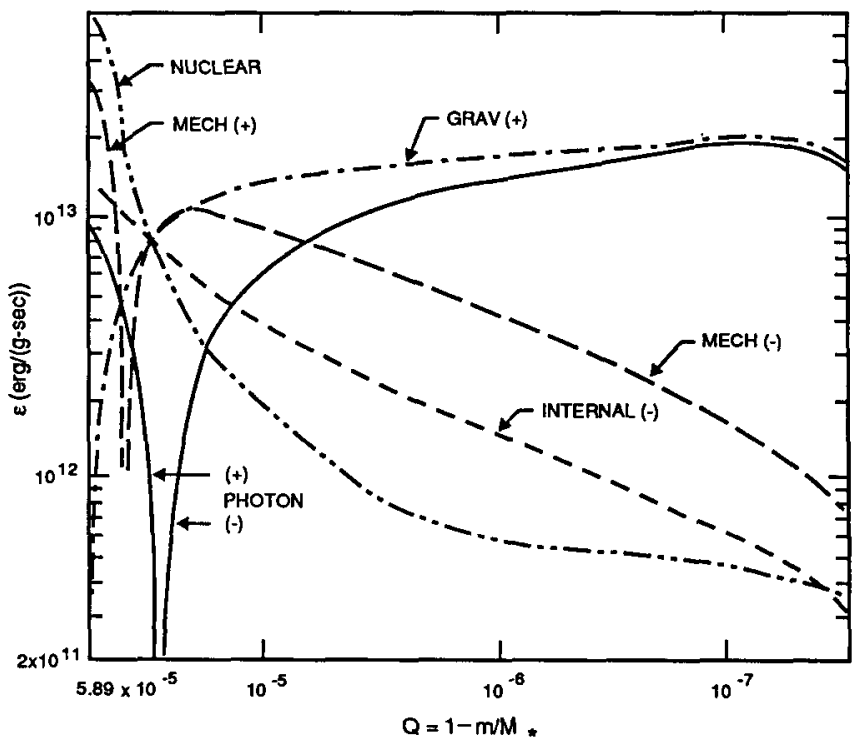

Fig. 4. Energy rates versus mass near peak nuclear burning on an accreting white dwarf. 


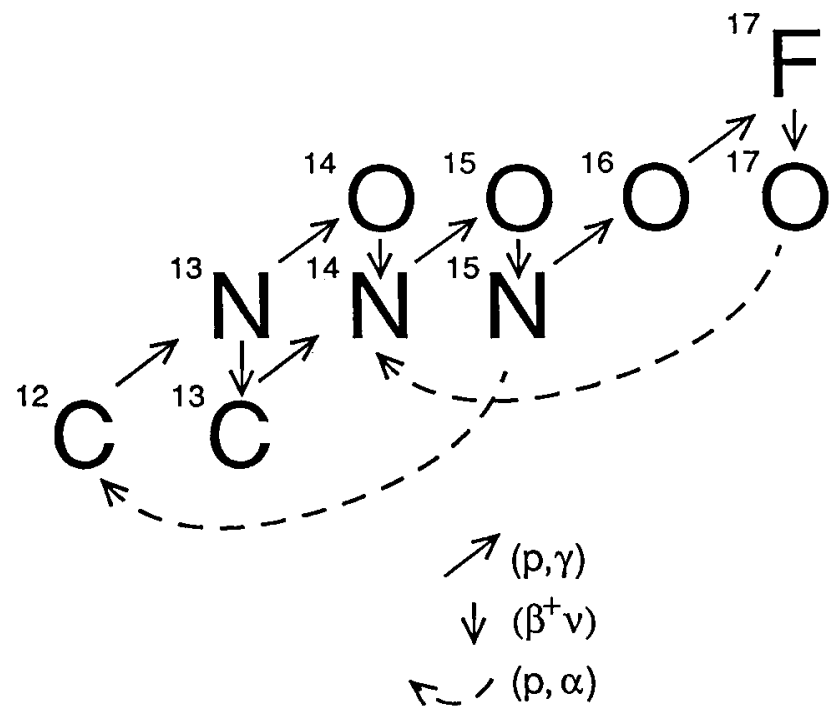

Fig. 5. The nuclei and reactions included in the network.

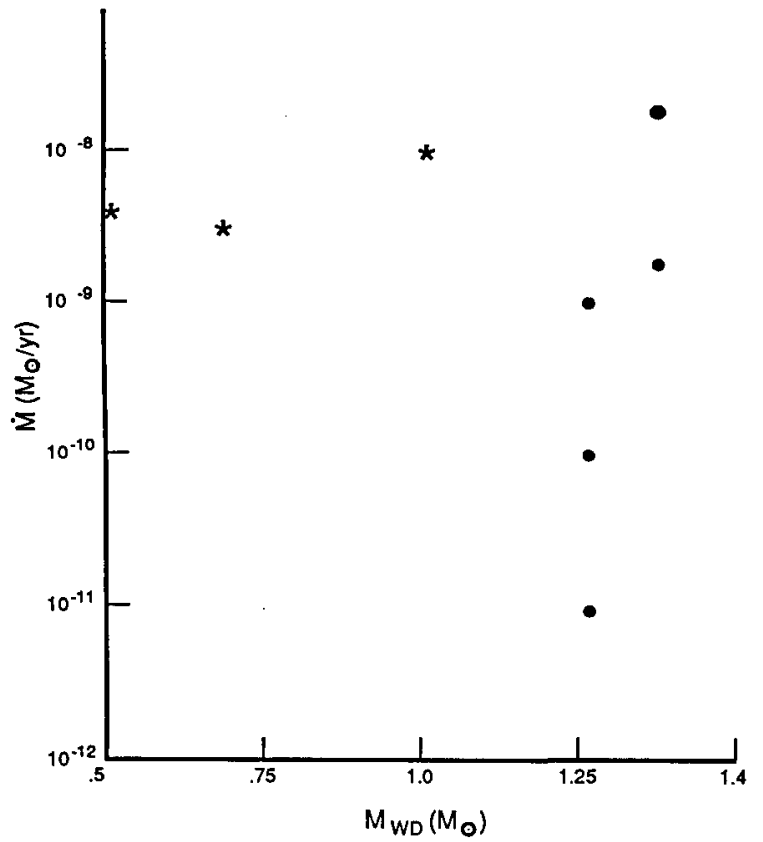

Fig. 6. Accretion rate versus white dwarf mass. Observed novae are indicated by asterisks and models by dots. 
89106652-9,adb

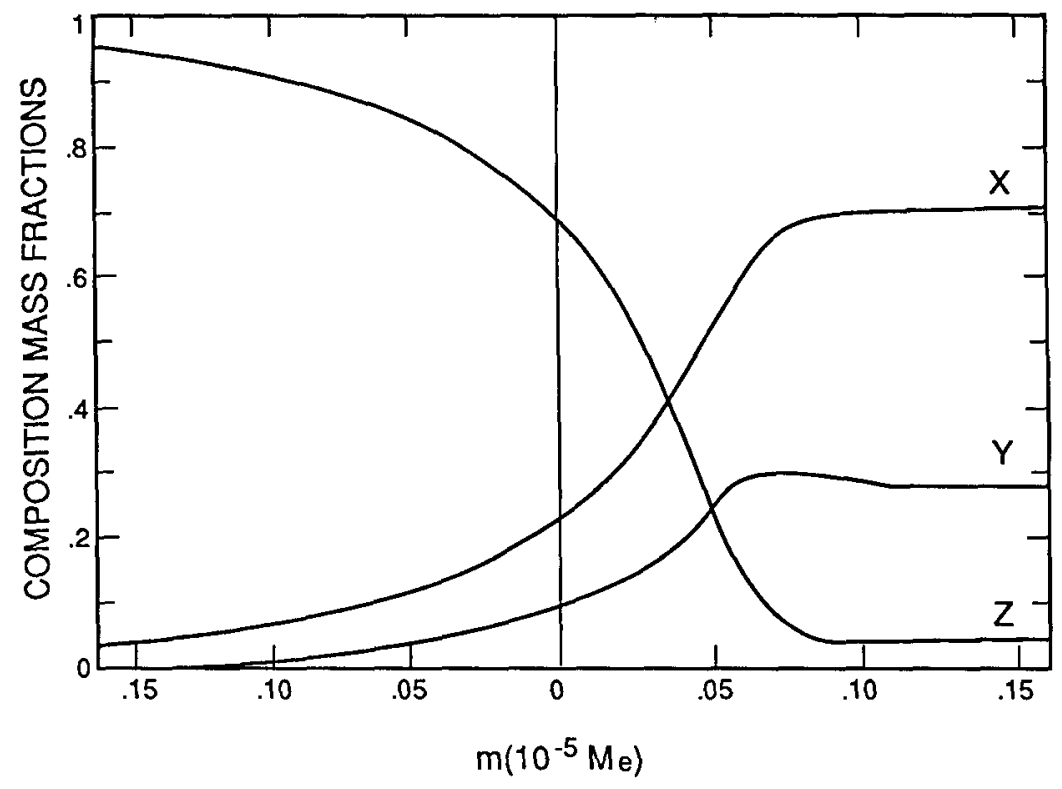

Fig. 7. Composition versus mass from Prialnik and Kovetz (1984).

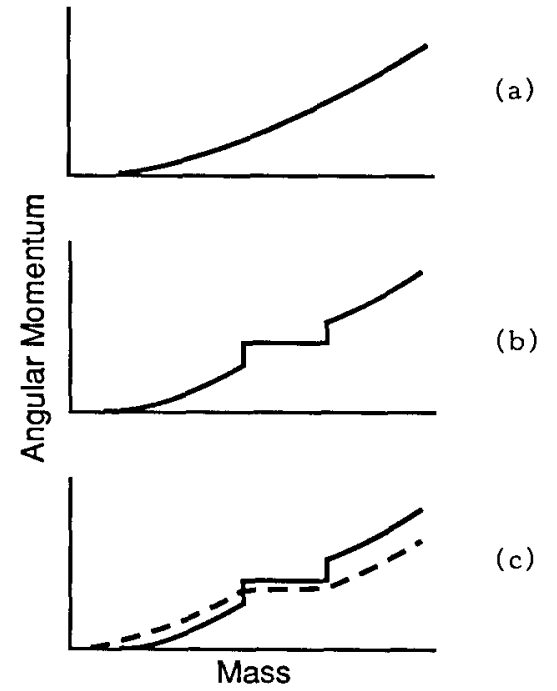

Fig. 8. Angular Momentum (a) before convection, (b) after convection, and (c) after convection-induced shear mixing. 